\title{
Correction to: Propositional Programs and Minimal Models
}

\section{Correction to:}

\section{Chapter 4 in: V. Lifschitz, Answer Set Programming, https://doi.org/10.1007/978-3-030-24658-7_4}

The book was inadvertently published with an incorrect format for table as

Table 4.1 Simplifying formulas containing logical constants

\begin{tabular}{l|l|l|l|l|l|l}
\hline Formula & $\neg \top$ & $\neg \perp$ & $F \wedge T$ & $F \wedge \perp$ & $F \vee \top$ & $F \vee \perp$ \\
\hline Simplification & $\perp$ & $\top$ & $F$ & $\perp$ & $\top$ & $F$ \\
\hline Formula & $\top \leftarrow F$ & $\perp \leftarrow F$ & $F \leftarrow \top$ & $F \leftarrow \perp$ & & \\
\hline Simplification & $\top$ & $\neg F$ & $F$ & $\top$ & & \\
\hline Set of formulas & $\Gamma \cup\{\top\}$ & $\Gamma \cup\{\perp\}$ & & & & \\
\hline Simplification & $\Gamma$ & $\perp$ & & & & \\
\hline
\end{tabular}

Whereas it should be

Table 4.1 Simplifying formulas containing logical constants

\begin{tabular}{|l|c|c|c|c|}
\hline Formula & $F \wedge \top$ & $F \wedge \perp$ & $F \vee \top$ & $F \vee \perp$ \\
\hline Simplification & $F$ & $\perp$ & $\top$ & $F$ \\
\hline
\end{tabular}

\begin{tabular}{|l|c|c|c|c|}
\hline Formula & $\top \leftarrow F$ & $\perp \leftarrow F$ & $F \leftarrow \top$ & $F \leftarrow \perp$ \\
\hline Simplification & $\top$ & $\neg F$ & $F$ & $\top$ \\
\hline
\end{tabular}

\begin{tabular}{|l|c|c|}
\hline Set of formulas & $\Gamma \cup\{T\}$ & $\Gamma \cup\{\perp\}$ \\
\hline Simplification & $\Gamma$ & $\perp$ \\
\hline
\end{tabular}

The updated online version of this chapter can be found at https://doi.org/10.1007/978-3-030-24658-7_4 